\title{
Index
}

A3f (Associazione 3 febbraio) 161, 171

activism 57, 158, 186

Alfasud 17, 159-161

Allied occupation 9, 16

see also black GIs; Four Days; military; occupation children

Almamegretta 131

alterity $67,85,109,130$

ambivalent 2, 39, 46-49, 51, 62-63, 71, 74, 76, $80,89,112,117,141-142,144,150,162$, 183-185, 186, 188-190

antihegemonic 10, 47, 132, 134, 158, 162, 169, 183

anti-immigration

politics 131,147

sentiment $61,63,74,111,139$

anti-racist $25,28,47-49,55-57,60,67,137$, 160-164, 169, 172, 175, 177, 179, 181-182

see also A3f; activism; Anti-Racist Forum;

Garibaldi 101; Lo Ska

Anti-Racist Forum 55, 174

Arabic 5, 26, 66, 72, 119

arte di arrangiarsi 24

Associazione 3 febbraio see A3f

austerity 3-4, 11, 39, 90, 97, 111, 153, 160, 162, 165,168

Babel 6-7, 39, 98, 186, 190

baby gangs 141-145

Bakhtin, Mikhail 40, 46, 53-55, 58, 60n1, 62, 72, $90,110,141,151,183$

see also carnivalesque; dialogic/dialogical; heteroglossia; speech genre(s);

Voloshinov, Valentin

banter 31, 66, 80, 90, 92-96, 100, 105-106, 112, $117,133,148-152$ barter 73, 108-109, 110, 115-126

Bassolino, Antonio 164

Beck, Ulrich 50

Belmonte, Thomas 38

belonging 6, 14, 22, 36, 40, 56, 58, 62-63, 64, 66, $79,82,85,106,115,126,133,190$

black GIs 16, 103

see also Allied occupation; occupation children

black masculinity 103, 139

Bourgois, Philippe 52, 110

Butler, Judith 48, 67

Buttitta, Ignazio 61-62

Camorra 24-25, 135-136, 164

see also mafia(s); organised crime

carnivalesque 46, 141, 151

Carosone, Renato 68-69

Casseri, Gianluca 139

catcalling 96-100

CGIL trade union 166-167, 171-172, 179

Chambers, Iain 11-13, 60, 97, 131-132

Chieke, Mbengue 139

Chinese merchandise 120-121, 126, 156

cholera 28, 134, 163-164

Cissè, Elhadji Diebel 139

City Hall 161-162, 171-174, 177-182

class

and language 15, 18, 39, 44

and masculinity 106

and politics 52, 110, 153, 160, 174, 188

see also postracialism

codes (linguistics) 44-45, 127

collective action 41, 53, 56, 160, 172

communication breakdown 63

competition 92, 120, 156-157 
contraband 23-25, 52, 82, 86, 116, 136, 147, 177 see also fake; hustling

convivial 45, 84, 86, 188

cosmopolitanism 43, 51, 73

counterpoetics 41,186

creolisation 12,41

crisis

and the economy 13, 76, 78, 128, 139, 156

and migration 49, 139, 174

as a narrative $3,162,165,183$

and rubbish 164

cultural meaning-making 11, 42, 58

cultural mediators 55, 81, 188

D’Alessandro, Libera 23, 25, 135-136

D’Amato, Pino 121

Daby and Sekou 139

Daniele, Pino 131

de Magistris, Mayor Luigi 4, 26, 161, 164, 169, 177, 181-182

death (talk and imaginaries of ) 162-171, 178,183

Decreto Sicurezza 3, 21

dexterity 39-40, 42-45, 50, 73, 94, 109, 127, 152,188

dialect 15, 22-23, 61-62, 80, 98, 117, 174

dialogic/dialogical 58-59, 79, 162, 167, 171, 178

see also Bakhtin, Mikhail; Voloshinov, Valentin

Diene, Idy 4, 139

Dieng, Moustapha 139

difference 6-7, 40-41, 62-63, 188, 190

diglossia 40, 45

Dines, Nick 22, 24-25, 51, 72, 161

Diop, Mame Mor 147

Diop, Mor 139

disease 16, 134, 163-166

see also cholera

Duchesca 82-83

earthquake (1981) 17, 29, 164

economic boom (post-war) 17-18

edginess 9, 41-42, 184, 188-189

emigration (from southern Italy) 12, 19-20, 22, 67-68, 71, 125, 131, 134

English (talk) 32-36, 68-72, 98, 114-115, 118-126, 189

everyday multiculture 188 fake $24,31,112,116,135-136,143,177,150$

see also contraband

far right 4, 140

fascism 14-16, 45-46, 61, 104

female morality 100

Fontanelle Cemetery 163

Four Days 16-17, 88

Fredrickson, George 188

Gaddafi, Muammar 2

Garibaldi 101 161, 172

gesture 38, 99, 105, 114, 146

Giuliani, Gaia 14, 103-104

Glissant, Edouard 6-7, 16, 40-42, 186 see also counterpoetics; multilingual talk; Relation

Gilroy, Paul 7-8, 49, 131

Goldberg, David Theo 49, 162, 174

Goraman 108-109

Gramsci, Antonio 12-16, 52-53

see also local-popular

Grand Tour 1, 13

greetings 58, 110-115

Gruppo Operaio '’E Zezi' 159-160

guappo 18, 43, 47, 80, 101, 116, 133, 140, 144, 174 see also masculinity

Gumperz, John 44

Hall, Stuart 52-53, 110

Hall, Suzanne 51

heteroglossia 53-58

heteronormative social order 94

Hewitt, Roger 45, 87, 90, 171

honour 80, 89, 101-103, 133

hooks, bell 48, 90

humour 45-48, 90, 106, 114, 117, 132, 148, 152, 176, 188

see also joking; laughter; swear words

hustling 52, 110, 129

Hymes, Dell 44-45

Iervolino, Rosa Russo 164

imperialism (Italian) 14

industry

contraband industry 116

Fordism in Napoli 17

tourist industry 24

infrapolitics 47, 132-133 
Italian Unification 12, 14, 47, 62, 130

Italiani brava gente 22

joking 72-74, 79, 95, 102, 139, 150, 190

see also humour; laughter; swear words

know-how 116, 126-128

knowing how to talk 76

Kunta, Kinte 76

language attitudes 18, 48-50, 62-63, 82, 86, 99 see also Smitherman, Geneva

language ideologies 15, 87 and figure of the native speaker 82

language use 6, 11-12, 17-22, 38-39, 58

laughter 82, 141, 151

see also humour; joking; swear words

learning new languages 72-76

legality

as ideology 4, 169, 185n1, 186

in street markets 136,177

Levi, Primo 8

linguistic veiling 47, 84, 178, 189

local-popular 53, 163-172, 183

local sexual conventions 94, 96, 101

Lombardi-Diop, Cristina 14

Lo Ska 55, 57

Louis Vuitton 31, 115

\section{Macerata 139}

'Made in Italy' 23-25, 32, 37n3, 119-120, 128, 153-136

see also merchandise

mafia(s) 24, 61, 120, 135, 140, 164

see also Camorra; organised crime

Malaparte, Curzio 16, 89

Mani pulite 164

market cries 110, 129

Marshall Plan 17

Masaniello's revolt (1647) 17

masculinity 13-14, 43, 80, 99, 103-106, 133, 139,150

see also guappo

Masslo, Jerry Essan 3, 140

Mbembe, Achille 2, 7-8, 11, 42, 163, 188

melancholia 48-50, 63

see also mourning

merchandise 25, 36, 47, 112, 114, 134-137, 145-149, 156 see also Chinese merchandise; 'Made

in Italy'

migration

anti-immigration politics 51, 63, 131, 147

anti-immigrant discourses 111, 125, 139

immigration legislation 3, 135

legal status 22, 110, 132, 135, 188

Mediterranean 2-3, 7-8, 11, 39, 42, 68, 92, 94, 106

migration to Italy 20-21

refugees 3-5, 137-139, 174

undocumented migrants 22, 135, 179

see also emigration (from southern Italy); racism

military 89-90, 96

miscegenation 84, 89, 105

Mitchell, Don 52

Modou, Samb 139

monolingual nationalism 50, 82, 87, 145

Monti, Mario 4, 157

Mor, Sogou 13

mourning 8, 71, 87

multilingual talk 6-9, 39, 52, 188

myfren 99, 112, 115-116

Napoli's Senegalese Association 161, 172, 179

Narducci, Giuseppe 170-171, 177, 181

NATO 68,88

Neapolitan language 39, 50, 56, 67

see also dialect

Niane, Magnane 147

not being able to talk 67,82

not being understood 67

not talking at all 50, 82

occupation children 89

opaque 1, 41-42, 83, 167, 190

organised crime 4, 134, 140

see also Camorra; mafia(s)

organised political action 160, 179

pacchisti 59, 89, 92

Pardo, Italo 127

Passerini, Luisa 45-46, 72, 90, 132

patriarchy 94,96

philo-Americanism 68

Piazza Garibaldi 4-5, 23-29, 72, 133, 137-138, $140,160-161,172,187$

Pine, Jason 18, 38-39, 43, 152 
Poggioreale market 32-36, 69-70, 91, 119, 125126, 136-137

police 24, 28-32, 145-152, 175-179

Polish (migrants) 76-78, 85

port city (Napoli) 18, 73

postracialism 49-50, 73

see also racial denial

Precari Bros 157, 160, 165, 171-172, 180

precariousness 14, 50-51, 71, 79, 109-110, 128, $132,153,164,166,172$

proletarian 17, 160-161

protest 51, 160, 169, 171-172, 179

public space(s) 79, 90, 100, 120, 137, 139, 164-165

public transport 36, 137

Pulcinella 38, 43, 47, 115

race management $24,161,173,189$

racial denial 174,190

racial intimacy $47,90,100-106$

racism $6-7,13,22,39,45,48,56-58,62,72-73$, 76, 89, 129n1, 167, 173-174, 177, 181, 188,190

racist formulae 132-134, 147-148

racist violence 3,140

Rampton, Ben 44, 82

Relation 58, 82-84, 90, 167, 190

see also Glissant, Edouard

respectability 39,94

Rettifilo 28, 31, 74, 187

Ricciardi, Franco 130-131

rights

migrant 24, 49, 50, 55-56, 164

talk $162,169,172-175$

risanamento 28

Roma 63, 134, 145, 187

rubbish 4, 153, 162-165

see also death (talk and imaginaries of ); disease

Rubin, Gayle 47, 90

Salvini, Matteo 3-4, 21, 139

Sanità 163

sapè fa 127-128

Sarnelli, Enrico 51, 118

Saviano, Roberto 134, 162

Schmoll, Camille 23-24, 51, 135

Schomberg, Jeff 52,110

Scott, James 47, 84, 132
Second World War 14, 17, 20-21, 47, 88, 91

Sementa, Luigi 177, 181

Senegalese migrants 135, 161

see also street vendors

Senese, James 89

Serao, Matilde 29

sex trafficking 94

sexual

conventions 47, 90-101

preserves 48

sexuality $47-48,90,94,104$

shops 26, 91, 119-120, 137, 148, 176

Simone, Abdoumaliq 7, 51, 188

Smitherman, Geneva 44, 49-50, 90, 133

Snowden, Frank 29, 134, 163

Southern Question 12-13

speaking back to power 163

speech genre(s) 40, 54-55, 58, 62, 98, 109, 134, 162

street vending 23-25, 37n3, 52, 110, 112, 129, 132-139, 150, 154, 158, 172, 187-188

mobile 85

unlicensed 4-5, 28, 32

unregulated 147

street vendors

anti-racism 56-57

Bangladeshi 98, 140-145

black 9, 109, 132-136, 140, 148, 177, 189

Chinese 84-85

Guinean 86

migrant 4-5, 51, 113-114, 137, 149, 150, 161,183

Neapolitan 4-5, 51, 72, 109, 118-120, 124, 153-158, 161, 169, 171, 174, 183

Senegalese 98, 105-106, 136

unlicensed 32, 92, 137, 145

Via Bologna 162-163

West-African 116, 135, 139, 145, 147, 174

women working as 91

Striscia la notizia 175-177

students 46, 160-161, 188

suicide $8,162,165-169$

swear words 56

tactics $45,87,111-112,128,141,148,171$

talking about talk 62

'Tammuriata nera' 88-89, 131

tourist(s) 4, 24, 31, 52, 74, 97-99, 161-167, 182-183, 187 
trade unions 160, 169

transcultural solidarity 82, 87, 132, 157, 181

translation 11, 59-60, 190

Trotter, Henry 43, 73, 110

Trumper, John 44

Tsing, Anna 1-2

u'maschiolona 43, 47, 93, 116

Ukrainian

language 72, 98, 119

migrants 22, 34, 81, 124

unemployment 7, 14, 18, 24-25, 38, 79, 160, 164,180

urban governance 164

urban regeneration 4

Vasto 5, 86, 111, 140

Verdicchio, Pasquale 12-13, 19-20, 53
Via Bologna 25-28, 57, 133, 161-163

the struggle for Via Bologna 169-185

Via Torino 166-169

victimhood 48-49, 67, 85

Viviani, Raffaele 107-108

Voloshinov, Valentin 53-54

see also Bakhtin, Mikhail

Vu' Cumprà 107-109

Wacquant, Loïc 52, 147

Ware, Vron 48, 90

wholesalers 23, 26

Wolof 26, 57, 75, 86-87, 98, 113, 179, 189

Zanotelli, Father Alex 183, 185n1 